\title{
Simulação numérica do comportamento não-linear de placas planas com perfurações quadradas sob compressão uniaxial em estruturas navais e offshore
}

\author{
Numerical simulation of the nonlinear behavior of flat plates with square holes under uniaxial \\ compression in naval and offshores structures. \\ B. S. Sentano ${ }^{1 *}$, M. de V. Real ${ }^{1}$, L. A. Isoldi ${ }^{1}$, L. G. Baptista ${ }^{1}$ \\ ${ }^{1}$ Escola de Engenharia, Universidade Federal do Rio Grande FURG, 96201-900, Rio Grande-RS, Brasil \\ *bruna.sentano@gmail.com
}

(Recebido em 22 de setembro de 2014; aceito em 29 de dezembro de 2014)

\begin{abstract}
Placas são elementos estruturais de superfície plana, cuja espessura é pequena quando comparada com as outras dimensões. Tem imensa aplicabilidade na engenharia devido ao seu desempenho estrutural quando solicitada à flexão, elevada relação resistência-peso e facilidade de descrição do seu comportamento mecânico devido aos eficientes métodos analíticos de cálculo. Estes tipos de estruturas podem ser delimitados por enrijecedores e constituem parte integrante de estruturas de navios, plataformas de petróleo offshore, comportas e docas flutuantes, por exemplo. Em estruturas navais, perfurações são comuns para a abertura de acessos, manutenção ou mesmo redução do seu peso total. Estas perfurações influenciam na máxima resistência desses elementos, pois ocorre uma alteração no comportamento mecânico da estrutura, devido à redistribuição das tensões ao longo da placa. Para o estudo do comportamento mecânico de placas com perfurações quadradas, foram realizadas análises inelásticas para a flambagem empregando-se o método dos elementos finitos. A amostra possui 120 placas em que a largura é mantida constante e o comprimento e espessura da placa e o lado do furo quadrado centralizado foram variados. A modelagem computacional foi realizada através do software ANSYS e foi possível determinar a carga última de resistência para cada caso, permitindo analisar a influência do tamanho do furo, da esbeltez e do comprimento da placa em sua capacidade de carga.
\end{abstract}

Palavras-chave: flambagem de placas, método dos elementos finitos, simulação numérica

Plates are structural elements of flat surface, whose thickness is small compared to other dimensions. These elements have wide applicability in engineering due to its structural performance when subjected to bending, high strength-weight ratio and ease description of the mechanical behavior due to the efficient analytical calculation methods. These types of structures can be delimited by stiffeners which form part of ships structures, oil offshore rigs, locks and floating docks, for example. In naval structures, holes are common to open access, maintenance or reducing the total weight. These holes influence on the ultimate strength of the plates, because they cause a change in mechanical behavior of the structure, due to the stress redistribution along the plate. Inelastic buckling analyses were performed to study the behavior of plates with square holes using the finite element method. The sample includes 120 plates where the width is kept constant and the length, the thickness and size of the central square hole are varied. The computational modeling was performed using the ANSYS software, and it was possible to determine the ultimate load resistance for each case, allowing to analyze the influence of hole size, slenderness aspect ratio of the plate in its load capacity.

Keywords: plate buckling, finite element method, numerical simulation.

\section{INTRODUÇÃO}

Placa plana é o elemento estrutural que possui a grandeza espessura muito menor que as suas outras dimensões. Tem imensa aplicabilidade na engenharia devido ao seu desempenho estrutural quando solicitada à flexão, além da elevada relação resistência-peso e capacidade de descrição de seu comportamento mecânico devido aos eficientes métodos analíticos de cálculo existentes.

De acordo com [1], placas planas limitadas por enrijecedores são partes integrantes das estruturas de navios, plataformas de petróleo offshore, comportas e docas flutuantes (Figura 1). As aberturas que são realizadas nas placas servem para o acesso e a manutenção ou 
simplesmente para a redução do peso total da estrutura e influenciam na máxima resistência desses elementos, pois o comportamento mecânico da estrutura se modifica devido à redistribuição das tensões ao longo da superfície placa.

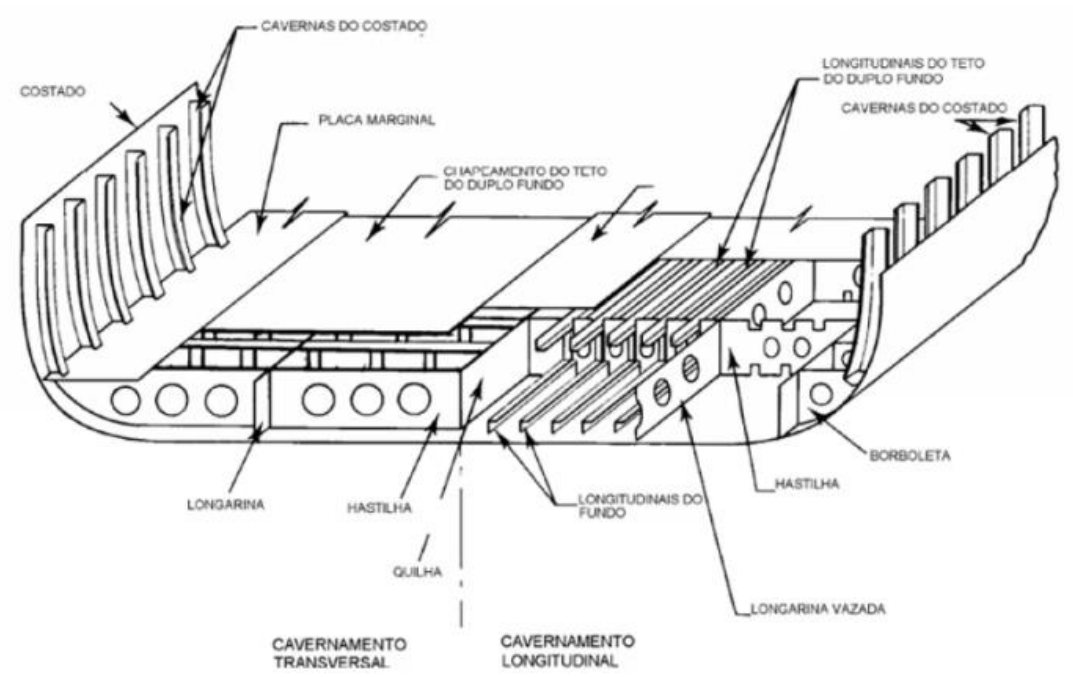

Figura 1: Estrutura terciária do duplo-fundo de um navio[2].

Em geral, estes elementos estruturais estão submetidos a carregamentos aplicados em seu próprio plano, conforme a Figura 2.

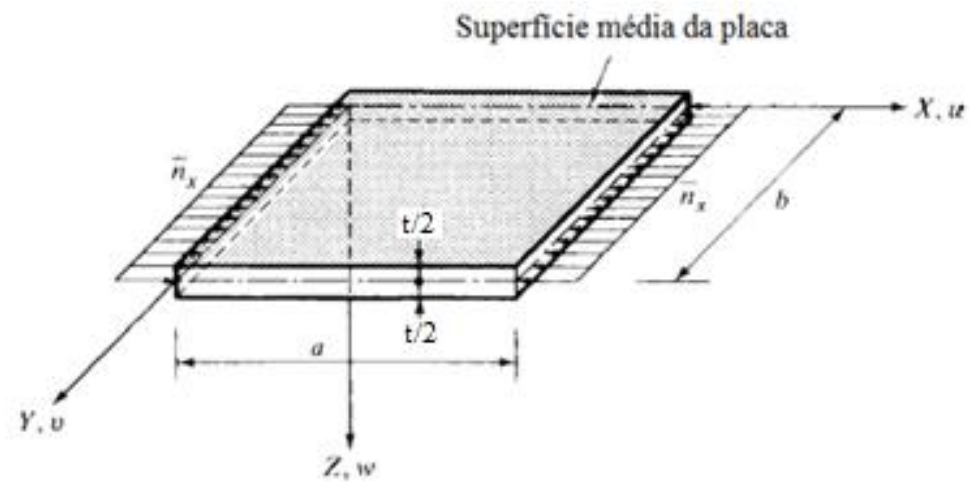

Figura 2: Placa retangular sob carregamento de compressão uniaxial aplicado no plano médio [3].

Forças de pequena intensidade aplicadas na superfície média causam deformação também no plano médio da placa, de tal forma que não há deslocamento lateral. Contudo, se a magnitude delas aumenta, o modo de deformação da placa se modifica, ou seja, a partir de um dado valor, as cargas causam deslocamentos transversais ao plano médio da placa. Assim, o equilíbrio que era estável para pequenos carregamentos torna-se instável quando a intensidade da carga se eleva. Esse fenômeno é denominado de flambagem e o valor da força que o gera é denominado de carga crítica. Quando a carga crítica é atingida, para qualquer incremento de força é produzido um acréscimo nos deslocamentos transversais, o que pode levar ao colapso da placa. A flambagem ocorre de maneira abrupta e dentro do regime elástico do material, ou seja, para tensões menores que a de escoamento, a estrutura atinge a ruptura.

Somente após 1930, com o desenvolvimento da indústria aeronáutica, é que as pesquisas apontaram que as placas planas possuem elevada resistência pós-flambagem [4]. Até esse período, quanto à flambagem, estimava-se que a falha de placas planas [5] e de barras compridas [6] era análoga. Entretanto, hoje se sabe que estruturas esbeltas, como as placas planas, apresentam a carga de ruptura maior que sua carga de flambagem. $\mathrm{O}$ carregamento extra que pode ser imposto às placas é de grande valia para a indústria naval e aeroespacial, pois ao se 
conceber o comportamento de pós-flambagem é possível encontrar elevada economia de peso estrutural.

O estudo de flambagem de placas teve início na observação do comportamento de placas planas de embarcações. A primeira proposta de solução para placas foi de [7] em 1883. Em 1891, [8] sugeriu a primeira solução de equação diferencial de placas, com a análise da tensão crítica elástica para uma placa retangular apoiada nos quatro bordos e sujeita a uma tensão uniforme de compressão longitudinal. Já [9] na análise elástica de placas, aumentou o número de condições de contorno. A análise inelástica começou com [10] em 1924 e desde então, a estabilidade de placas tem sido pesquisada e estudada por vários autores. Entre os pesquisadores, [11] desenvolveram experimentalmente gráficos que apresentam a capacidade de resistência à compressão uniaxial de placas com furos quadrados e circulares. Em [12] foram estudados os efeitos de furos em placas com enrijecedores sob compressão uniaxial, biaxial e cisalhamento puro. Em [13] foi empregado o Método dos Elementos Finitos na análise de flambagem inelástica de placas quadradas com furos centrais de geometria quadrada e circular. Em [14] foram apresentadas formulações para a resistência última de chapas de navios sob uma combinação de tensões de compressão uniaxial, cisalhamento no bordo e cargas de compressão lateral. Já em [15] foi aplicado o Método dos Elementos Finitos para estabelecer a tensão de flambagem elastoplástica de placas quadradas e retangulares simplesmente apoiadas com furo circular e sob carregamento uniaxial. Em [16] foi estudado o efeito da dimensão do furo e a localização da flambagem no modo elástico de placas retangulares submetidas a carregamento de compressão uniaxial uniforme. Por fim, em [17] foi estudado o comportamento inelástico de pós-flambagem de placas quadradas e retangulares, com furos circulares centrados sob compressão uniaxial em estruturas navais e offshore.

As respostas encontradas através dos estudos teóricos de placas finas foram corroboradas através de pesquisas que empregam a solução numérica e a aplicação de métodos de análise computacional de placas.

Logo, com base no método dos elementos finitos e empregando a modelagem computacional através do software ANSYS, o objetivo deste artigo é a realizar a análise não-linear da flambagem de placas planas, compostas de material isotrópico e homogêneo, submetida à carregamento mecânico uniaxial de compressão no próprio plano. $\mathrm{O}$ estudo estende-se à influência dos furos, espessura e comprimento da placa na capacidade de carga de uma amostra de 120 placas planas que possuem ou não perfurações quadradas centrais.

\section{METODOLOGIA}

A simulação numérica foi realizada no software ANSYS, que é um programa computacional empregado em inúmeros problemas de engenharia e é baseado no Método dos Elementos Finitos. A análise por elementos finitos possibilita modelar o comportamento não-linear do material e a geometria da placa. A modelagem da placa inicia-se com a definição da geometria da região de interesse. Para isso, gera-se um retângulo de dimensões a $\times b \mathrm{~m}$ da placa. $\mathrm{O}$ furo é criado a partir de um quadrado de dimensão $\mathrm{d} \times \mathrm{d} \mathrm{m}$ com a origem determinada pelos parâmetros a e b da placa e d do furo (Figura 3). Assim, as coordenadas da origem do furo são definidas por: $o=\left(\frac{a}{2}-\frac{d}{2}, \frac{b}{2}-\frac{d}{2}\right)$.

A divisão do domínio é compatível com o tamanho dos elementos, e a malha é gerada no comando "Size Controls", disponível no menu "Meshing" em "Mesh Tool". Na região do furo, em que há concentração de tensões, e por isso é de interesse para análise, é realizado o refinamento da malha, como pode ser observado na Figura 4. A placa é constituída de material linear elástico perfeitamente plástico e geometria inicial imperfeita, em que o modo de flambagem de uma pré-análise de autovalor elástico é admitida. 


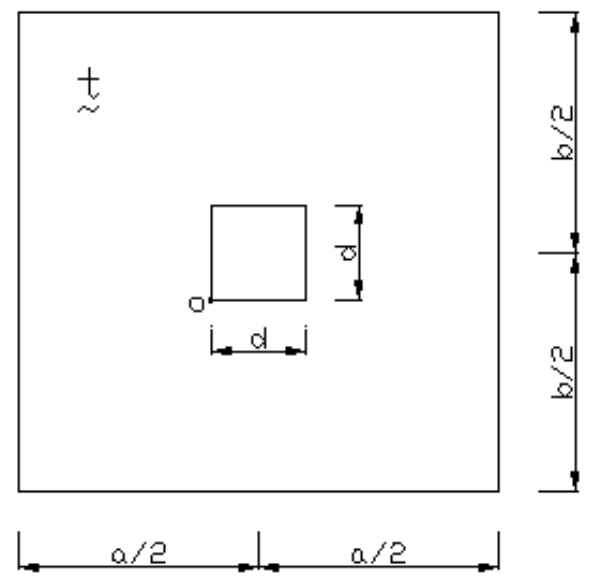

Figura 3: Placa plana de aço com furo quadrado centrado.

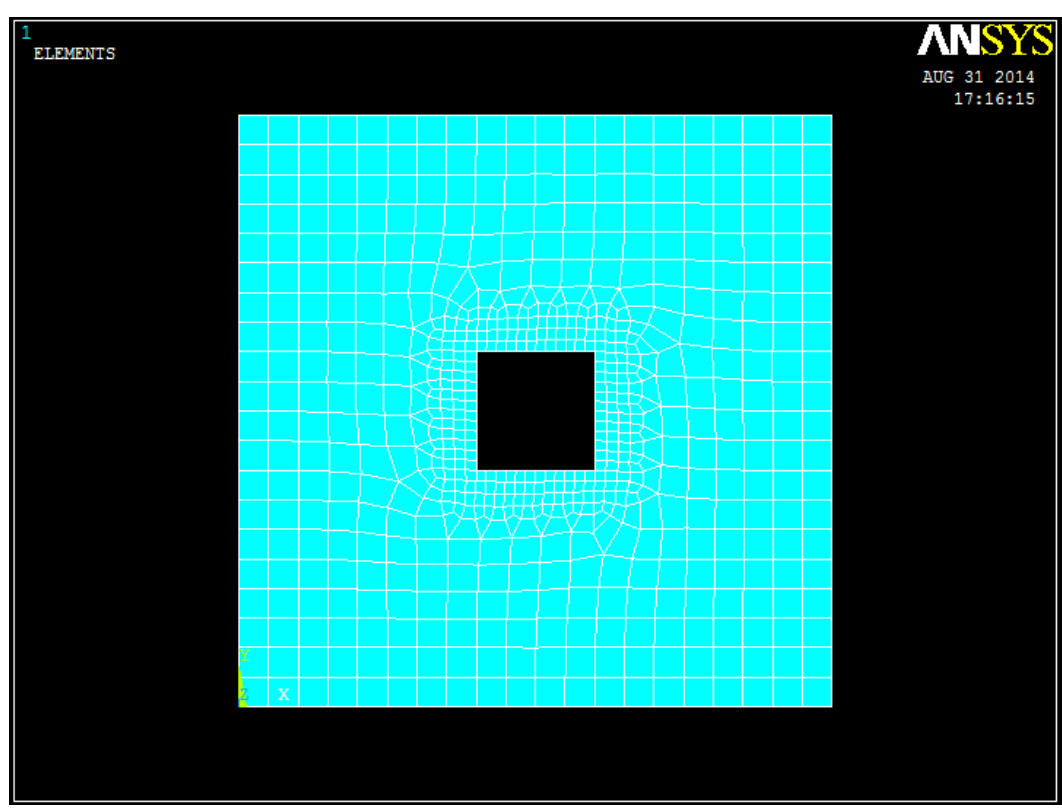

Figura 4: Malha de elementos finitos de uma placa plana de aço com furo quadrado centrado.

Como indicado na Figura 5, os quatro bordos das placas são considerados como simplesmente apoiadas e todos os nós ao longo de todas as bordas são restringidas à deflexão e rotação ao longo da espessura $(\mathrm{Uz}, \mathrm{Rz}=0)$. As bordas descarregadas podem deformar no plano, mas continuam em linha reta, pois o acoplamento faz com que os deslocamentos sejam uniformes ao longo do comprimento da placa. Esta condição possibilita simular a situação em que a placa conta com reforços longitudinais. Na borda da placa restringida $(\mathrm{Ux}=0)$, surge uma força causada pelo carregamento da borda.

Pesquisando o tipo de elemento que apresente resultado com acurácia e rapidez, foram designados dois tipos de elementos do software ANSYS, o SHELL181 e o SHELL93. O elemento do tipo SHELL foi escolhido, pois possui aplicabilidade na modelagem de estruturas finas, ou seja, estruturas em que a espessura é muito pequena em relação às outras dimensões. $O$ elemento SHELL181 apresenta quatro nós, onde cada nó possui seis graus de liberdade, três de rotação e três de translação. Já o elemento SHELL93 tem oito nós, onde cada nó possui seis graus de liberdade, três de rotação e três de translação. Segundo [15], o valor máximo da imperfeição é determinado pela expressão $w 0=b / 2000$, onde b é a largura da placa em metros. Os testes de convergência de malha foram aplicados em uma placa quadrada com furo quadrado centrado que apresenta características que estão apontadas na Tabela 1. 


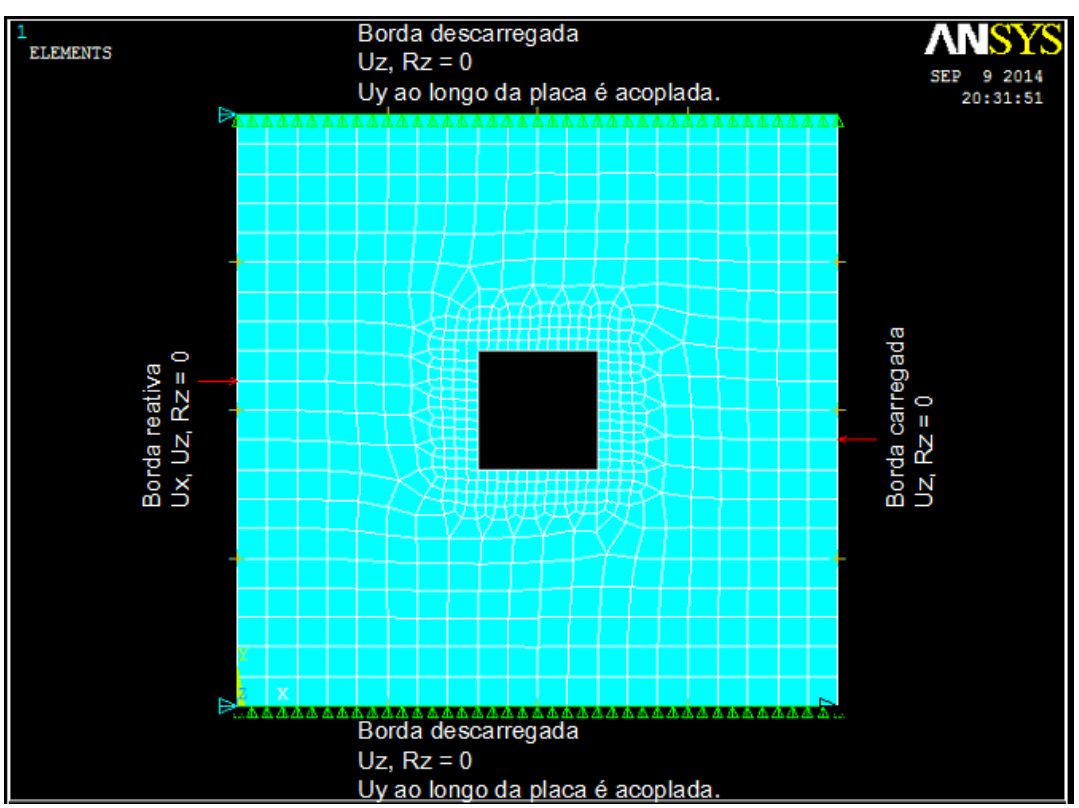

Figura 5: Condições de contorno para a modelagem de placas.

Tabela 1: Teste de convergência do elemento: Características da placa modelo.

\begin{tabular}{cccccccc}
\hline $\begin{array}{c}\mathrm{a}=\mathrm{b} \\
\left(10^{-3} \mathrm{~m}\right)\end{array}$ & $\begin{array}{c}\mathrm{t} \\
\left(10^{-3} \mathrm{~m}\right)\end{array}$ & $\begin{array}{c}\mathrm{d} \\
\left(10^{-3} \mathrm{~m}\right)\end{array}$ & $\mathrm{d} / \mathrm{b}$ & $\mathrm{b} / \mathrm{t}$ & $\begin{array}{c}\mathrm{w} 0 \\
\left(10^{-6} \mathrm{~m}\right)\end{array}$ & $\begin{array}{c}\mathrm{E} \\
(\mathrm{GPa})\end{array}$ & $\sigma y$ \\
\hline 125 & 1,615 & 25 & 0,2 & 77,4 & 9,7 & 210 & 323,3 \\
\hline
\end{tabular}

Os resultados obtidos da simulação, em que o tamanho do elemento varia, encontram-se na Tabela 2 .

Tabela 2: Teste de convergência dos elementos SHELL93 e SHELL181.

\begin{tabular}{ccccccc} 
& & & \multicolumn{2}{c}{ SHELL93 } & \multicolumn{2}{c}{ SHELL181 } \\
\cline { 3 - 6 } Teste & $\begin{array}{c}\text { Tamanho do } \\
\text { elemento } \\
\left(10^{-3} \mathrm{~m}\right)\end{array}$ & Malha & $\begin{array}{c}\text { P, ANSYS } \\
(\mathrm{kN})\end{array}$ & $\begin{array}{c}\text { Diferença } \\
\text { Relativa }(\%)\end{array}$ & $\begin{array}{c}\text { P, ANSYS } \\
(\mathrm{kN})\end{array}$ & $\begin{array}{c}\text { Diferença } \\
\text { Relativa (\%) }\end{array}$ \\
\hline 1 & 25 & 5 & 34,2541 & 0,705 & 35,4634 & 3,642 \\
2 & 12,5 & 10 & 34,0584 & 0,129 & 34,7688 & 1,612 \\
3 & 6,25 & 20 & 34,0101 & 0,013 & 34,3805 & 0,477 \\
4 & 3,125 & 40 & 34,0144 & 0,000 & 34,2173 & 0,000 \\
\hline
\end{tabular}

Observa-se que a diferença relativa para as malhas mais refinadas é menor para o elemento SHELL93 do que para o elemento SHELL181, e por isso será o tipo de elemento adotado para o modelo. Quanto ao tamanho da malha, foram realizados testes para dois tipos de placas. Os dados que caracterizam as placas estão contidos na Tabela 3.

Tabela 3: Características geométricas das placas modelo para o teste de malha.

\begin{tabular}{cccccccc}
\hline Placa & $\begin{array}{c}\mathrm{a}=\mathrm{b} \\
\left(10^{-3} \mathrm{~m}\right)\end{array}$ & $\mathrm{t}\left(10^{-3} \mathrm{~m}\right)$ & $\mathrm{d}\left(10^{-3} \mathrm{~m}\right)$ & $\mathrm{d} / \mathrm{b}$ & $\mathrm{w} 0\left(10^{-3} \mathrm{~m}\right)$ & $\mathrm{E}(\mathrm{GPa})$ & $\sigma \mathrm{y}(\mathrm{Mpa})$ \\
\hline $\mathrm{A}$ & 125 & 1,615 & 0 & 77,4 & 0,229 & 210 & 323,3 \\
$\mathrm{~B}$ & 125 & 1,615 & 25 & 77,4 & 0,097 & 210 & 323,3 \\
\hline
\end{tabular}

Os resultados obtidos estão apresentados em Tabela 4 e Figura 6. 
Tabela 4: Teste de convergência de malha.

\begin{tabular}{ccccccccccc} 
& & & & \multicolumn{3}{c}{ PLACA A } & \multicolumn{3}{c}{ PLACA B } \\
\cline { 3 - 10 } Teste esize & $\begin{array}{c}\text { Tamanho } \\
\text { do } \\
\text { elemento } \\
\left(10^{-3} \mathrm{~m}\right)\end{array}$ & & & $\begin{array}{c}\text { Número } \\
\text { Me }\end{array}$ & $\begin{array}{c}\text { P, ult } \\
\text { Ansys } \\
(\mathrm{kN})\end{array}$ & $\begin{array}{c}\text { Diferença } \\
\text { Relativa } \\
(\%)\end{array}$ & $\begin{array}{c}\text { Número } \\
\text { de } \\
\text { Elementos }\end{array}$ & $\begin{array}{c}\text { P, ult } \\
\text { Ansys } \\
(\mathrm{kN})\end{array}$ & $\begin{array}{c}\text { Diferença } \\
\text { Relativa } \\
(\%)\end{array}$ \\
\hline 1 & $\mathrm{~b} / 5$ & 25 & 5 & 5 & 136 & 38,04 & 0,799 & 104 & 34,25 & 0,705 \\
2 & $\mathrm{~b} / 10$ & 12,5 & 10 & 10 & 195 & 37,75 & 0,030 & 264 & 34,06 & 0,129 \\
3 & $\mathrm{~b} / 20$ & 6,25 & 20 & 20 & 584 & 37,71 & $-0,086$ & 644 & 34,01 & $-0,013$ \\
4 & $\mathrm{~b} / 40$ & 3,125 & 40 & 40 & 1899 & 37,74 & 0,000 & 1997 & 34,02 & 0,000 \\
\hline
\end{tabular}

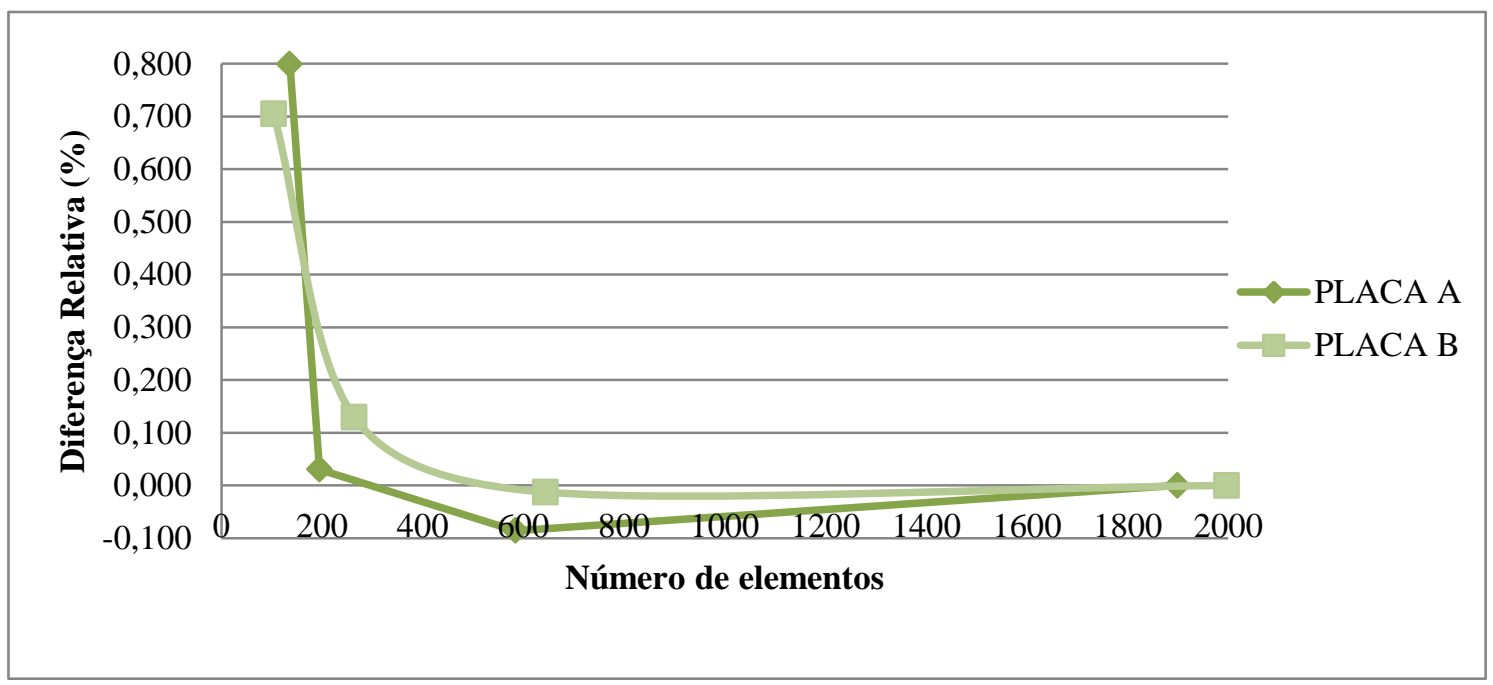

Figura 6: Teste de convergência.

Observa-se que, quanto maior for o refinamento da malha, melhor serão os resultados obtidos, porém o tempo de processamento da solução também é maior. Os resultados encontrados no Teste 4 foram considerados na determinação da diferença relativa. Nota-se que o Teste 4 foi o mais preciso na solução do problema. Entretanto, o Teste 3 apresentou resultados com precisão satisfatória e tempo de processamento menor que o Teste 4. Logo, o tamanho da malha adotado para o presente artigo foi a que possui elementos com tamanho b/20.

Foram considerados os resultados experimentais obtidos em [11] para a validação do modelo. O estudo de [11] prevê a capacidade de carga máxima e o comportamento pós-flambagem de placas perfuradas. Na Tabela 5 são apresentados os resultados dos testes experimentais elaborados por [11].

Tabela 5: Valores experimentais obtidos por [11].

\begin{tabular}{cccccccccc}
\hline PLACA & $\begin{array}{c}\mathrm{a}=\mathrm{b} \\
\left(10^{-3} \mathrm{~m}\right)\end{array}$ & $\begin{array}{c}\mathrm{T} \\
\left(10^{-3} \mathrm{~m}\right)\end{array}$ & $\begin{array}{c}\mathrm{d} \\
\left(10^{-3} \mathrm{~m}\right)\end{array}$ & $\mathrm{d} / \mathrm{b}$ & $\mathrm{b} / \mathrm{t}$ & $\begin{array}{c}\mathrm{W}_{0} \\
\left(10^{-3} \mathrm{~m}\right)\end{array}$ & $\mathrm{E}(\mathrm{GPa})$ & $\begin{array}{c}\sigma \mathrm{y} \\
(\mathrm{MPa})\end{array}$ & $\begin{array}{c}\mathrm{P}, \mathrm{ult} \\
(\mathrm{kN})\end{array}$ \\
\hline PL1A & 125 & 1,615 & 0 & 0 & 77,40 & 0,229 & 210 & 323,3 & 39,32 \\
SQ2 & 125 & 1,615 & 25 & 0,2 & 77,40 & 0,097 & 210 & 323,3 & 33,48 \\
SQ3 & 125 & 1,615 & 37,5 & 0,3 & 77,40 & 0,141 & 210 & 323,3 & 28,85 \\
SQ4 & 125 & 1,615 & 50 & 0,4 & 77,40 & 0,113 & 210 & 323,3 & 25,52 \\
SQ5 & 125 & 1,615 & 62,5 & 0,5 & 77,40 & 0,209 & 210 & 323,3 & 21,86 \\
\hline
\end{tabular}

Nos ensaios elaborados por [11], os nós nos lados não carregados não foram restringidos e, por isso o acoplamento não é considerado na validação do modelo. A validação do modelo será 
através da comparação das cargas máximas obtidas pelo software ANSYS com a solução experimental, como é visto na Tabela 6.

Tabela 6: Comparação dos resultados.

\begin{tabular}{ccccc}
\hline PLACA & $\begin{array}{c}\text { Pult,exp } \\
(\mathrm{kN})\end{array}$ & $\begin{array}{c}\text { Pult,ANSYS } \\
(\mathrm{kN})\end{array}$ & $\begin{array}{c}\text { Pult,ANSYS/ } \\
\text { Pult,exp }\end{array}$ & $\begin{array}{c}\text { Diferença } \\
\text { Relativa (\%) }\end{array}$ \\
\hline PL1a & 39,32 & 35,11 & 0,89 & 10,71 \\
SQ2 & 33,48 & 34,01 & 1,02 & $-1,58$ \\
SQ3 & 28,85 & 31,88 & 1,11 & $-10,50$ \\
SQ4 & 25,52 & 29,36 & 1,15 & $-15,05$ \\
SQ5 & 21,86 & 26,44 & 1,21 & $-20,95$ \\
\hline
\end{tabular}

Conclui-se que o modelo numérico que emprega o Método dos Elementos Finitos modela o comportamento mecânico e prevê a capacidade de carga de placas perfuradas com precisão satisfatória sob o ponto de vista da engenharia, visto que o valor médio da razão Pult,ANSYS/Pult,exp é de 1,07 e o coeficiente de variação de 0,1106.

Já quanto à geometria das placas, o estudo feito neste artigo contempla placas planas quadradas e retangulares, com ou sem perfuração quadrada centrada, submetidas à compressão uniaxial, em que onde a é o comprimento e $\mathrm{b}$ a largura, ambas medidas em metros.

Segundo [14], o índice de esbeltez $\beta$ é determinado pela Equação (1):

$$
\beta=\frac{b}{t} \sqrt{\frac{E}{\sigma_{y}}}
$$

onde: $\mathrm{t}$ é a espessura da placa em metros, $\mathrm{d}$ a aresta do furo em metros, $\mathrm{E}$ o módulo de elasticidade do aço igual a $210 \mathrm{GPa}$. As placas são compostas pelo aço AH36 que possui tensão de escoamento $\sigma \mathrm{y}=355 \mathrm{MPa}$.

As simulações foram distribuídas em seis agrupamentos, em que cada um apresenta variações paramétricas no tamanho e espessura da placa e no tamanho do furo. Os parâmetros encontramse nas Tabelas 7,8 e 9.

Tabela 7: Variações paramétricas em relação ao comprimento da placa.

\begin{tabular}{cccc}
\hline GRUPO & $\mathrm{a}\left(10^{-3} \mathrm{~m}\right)$ & $\mathrm{b}\left(10^{-3} \mathrm{~m}\right)$ & $\mathrm{a} / \mathrm{b}$ \\
\hline 1 & 900 & 900 & 1 \\
2 & 1800 & 900 & 2 \\
3 & 2700 & 900 & 3 \\
4 & 3600 & 900 & 4 \\
5 & 4500 & 900 & 5 \\
6 & 5400 & 900 & 6 \\
\hline
\end{tabular}

Tabela 8: Variações paramétricas em relação à espessura da placa.

\begin{tabular}{ccc}
\hline $\mathrm{t}\left(10^{-3} \mathrm{~m}\right)$ & $\beta$ & $\mathrm{b} / \mathrm{t}$ \\
\hline 10 & 2,19 & 90 \\
12,5 & 1,75 & 72 \\
15 & 1,46 & 60 \\
19 & 1,15 & 47,37 \\
22 & 0,99 & 40,91 \\
\hline
\end{tabular}


Tabela 9: Variações paramétricas em relação à aresta do furo.

\begin{tabular}{cc}
\hline $\mathrm{d}\left(10^{-3} \mathrm{~m}\right)$ & $\mathrm{d} / \mathrm{b}$ \\
\hline 0 & 0 \\
180 & 0,2 \\
350 & 0,4 \\
540 & 0,6 \\
\hline
\end{tabular}

\section{RESULTADOS E DISCUSSÃO}

A Figura 7 apresenta as tensões normais médias obtidas através da divisão da força última determinada pelo software ANSYS pela área da seção transversal da placa bxt e as deformações normais médias na direção $\mathrm{x}(\varepsilon \mathrm{x})$, decorrente dos incrementos de deslocamento $\mathrm{Ux}$ ao longo da borda carregada em $\mathrm{x}=0$, para a placa cuja relação $\mathrm{a} / \mathrm{b}=1 \mathrm{e} \mathrm{t}=10 \times 10-3 \mathrm{~m}$, com um único furo central quadrado, no qual o tamanho do furo é variado. Segundo [14], o índice de esbeltez de placas de navios e estruturas offshore é de 1,50 a 3,50.

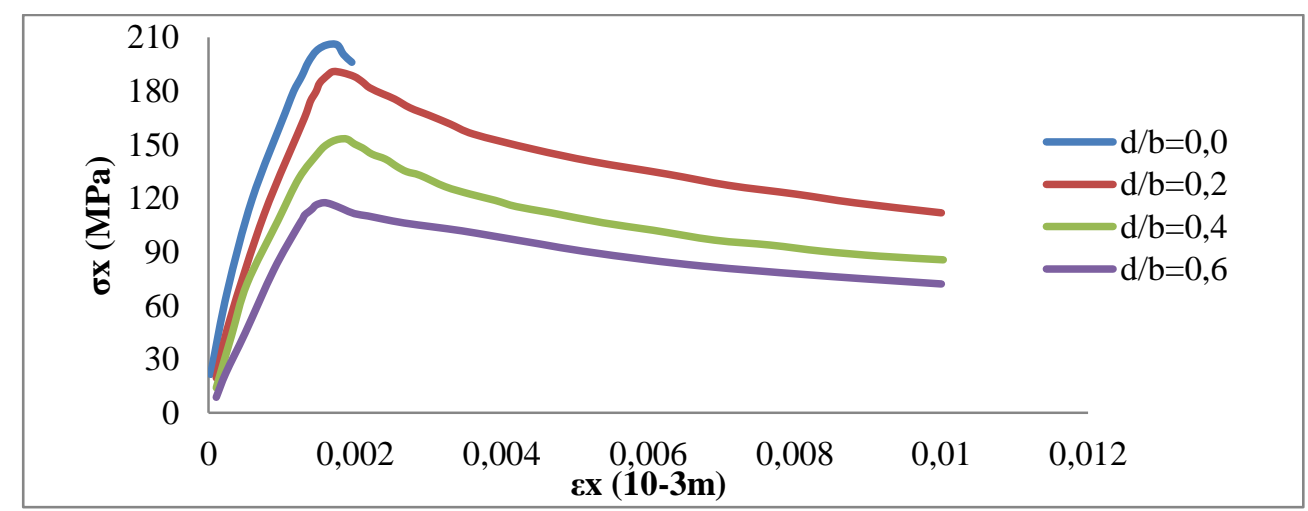

Figura 7: Influência do furo para a placa com relação a/b $=1$ e $t=10 \times 10^{-3} \mathrm{~m}$.

Percebe-se que a carga de ruptura de flambagem diminui à medida que o tamanho do furo aumenta, ou seja, quando a área do orifício quadrado corresponde a $36 \%$ da área total da placa $(d / b=0,6)$ a força última é reduzida em $42,88 \%$ quando comparada com a placa sem furo.

Conforme a Figura 8, a carga de ruptura de uma placa sofre grande influência de sua esbeltez. Observa-se que, independentemente da geometria, quanto menor a relação b^t e mais espessa a placa, maior será a sua resistência. Verifica-se também que, independentemente da espessura da placa, ao passo que o furo aumenta, as tensões normais médias convergem. Isso se deve ao fato, de que a carga última limitar-se à tensão normal de escoamento do material $\sigma y$.

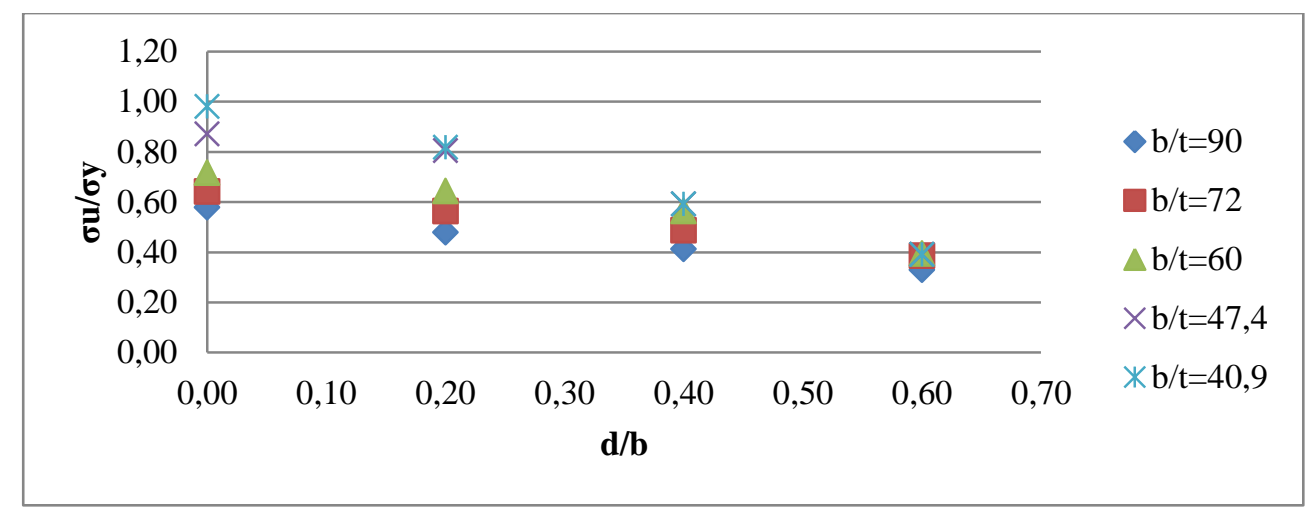

Figura 8: Efeito da esbeltez quando a relação $a / b=2$. 
Na Figura 9 são apresentados os resultados obtidos dos testes em que ocorre a variação no parâmetro de proporção $a b$ das placas.

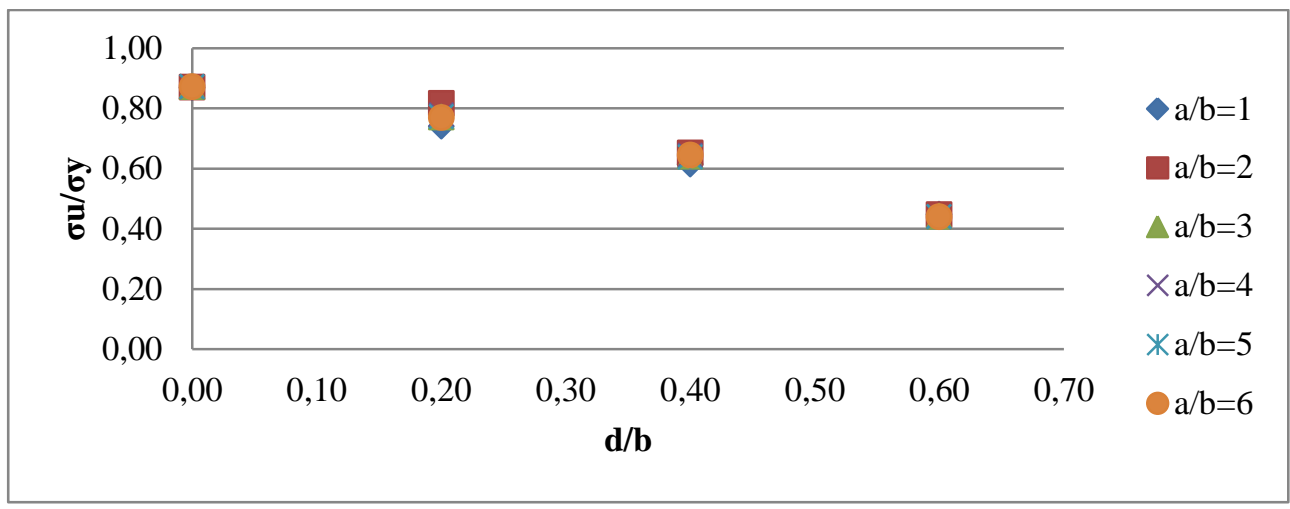

Figura 9: Variação no parâmetro de proporção quanto $t=19 \times 10^{-3} \mathrm{~m}$.

Observa-se que a resistência última da placa não sofre influência significativa da variação da relação a/b. Logo, conclui-se que quanto maior a espessura da placa, menor são as variações na resistência última.

\section{CONCLUSÃO}

Foram verificados a influência do tamanho do furo, o efeito da esbeltez da placa e a relação a/b na capacidade de carga de uma amostra que contém 30 placas sem perfuração e 90 com furo quadrado central. As placas analisadas estão sujeitas à compressão uniaxial uniforme. $\mathrm{O}$ estudo apontou que, placas planas sem furos apresentam resistência última superior às placas perfuradas. Quanto ao furo, a capacidade de carga da placa diminui à medida que a perfuração aumenta. Bem como, mesmo com o aumento do furo, ocorre a convergência da tensão última, independentemente do índice de esbeltez da placa. Verifica-se ainda que quanto mais espessa a placa, maior será a sua resistência. Já a análise da proporção entre os lados $a$ e $b$ da placa, mostrou que a resistência última da placa não sofre alteração significativa.

\section{REFERÊNCIAS BIBLIOGRÁFICAS}

1. Kumar MS. Ultimate strength of square plate with rectangular opening under axial compression. $\mathbf{J}$ Naval Architec Marine Engine. 2007 Jun;4:15-26.

2. Pinto S. Elementos estruturais de navios. Rio de Janeiro; 2011.

3. Szilard R. Theories and applications of plate analysis - classical numerical and engineering methods. New Jersey: John Wiley \& Sons; 2004. 1024 p.

4. Schuman L. Back G. Strength of rectangular plates under edge compression. NASA Technical Report, TR, R-40, 1959.

5. Roorda J. Buckling of elastic structures. Waterloo: University of Waterloo Press; 1980. 123 p.

6. Trahair NS, Bradford MA. The behavior and design of steel structures. New York: E \& FN Spon; 1988. $420 \mathrm{p}$.

7. Clebsch A, Saint-Venant AJCBD. Théorie de l'elasticité des corps solides. Paris: Dunod; 1883. 900 p.

8. Bryan GH. On the stability of a plane under thrusts in its own plane with applications to the buckling of the sides of a ship. Proc London Math Soc. 1891;22(1):54-67, doi:10.1112/plms/s122.1.54.

9. Timoshenko SP. Einige stabilitäts probleme der elastizitäts theorie. Z Math Phys. 1910;58(4).

10. Bleich F. Theorie und berechnung der eisernen brücken. Berlim: Verlag Von Julius Springer; 1924, doi:10.1007/978-3-642-99614-6.

11. Narayanan R, Chow FY. Ultimate capacity of uniaxially compressed perforated plates. Thin Wall Struct. 1984 Mar;2(3):242-64, doi:10.1016/0263-8231(84)90021-1.

12. Shanmugam NE. Openings in thin-walled steel structures. Thin Wall Struct. 1997 Jul;28(3-4)355-72, doi:10.1016/S0263-8231(97)00053-0. 
13. Shanmugam NE, Thevendran V, Tan YH. Design formula for axially compressed perforated plates. Thin Wall Struct. 1999 May;34(1)1-20, doi:10.1016/S0263-8231(98)00052-4.

14. Paik JK, Thayamballi AK, Kim BJ. Advanced ultimate strength formulations for ship plating under combined biaxial compression/tension, edge shear, and lateral pressure loads. Mar Technol. 2001 Jan;38(1)9-25, doi:0025-3316/01/3801-0009\$00.59/0

15. El-Sawy KM, Nazmy AS, Martini MI. Elasto-plastic buckling of perforated plates under uniaxial compression. Thin Wall Struct. 2004 Aug;42(8):1083-101, doi:10.1016/j.tws.2004.03.002.

16. Real MV, Isoldi LA. Effect of circular holes dimension and location on the elastic buckling load of rectangular plates. In: 21st Brazilian Congress of Mechanical Engineering, p. 24-28, 2011.

17. Baptista LG. Simulação numérica não-linear do comportamento pós-flambagem de placas finas de aço sob compressão uniaxial em estruturas navais e offshore. Dissertação (Mestrado em Engenharia Oceânica) - Programa de Pós-Graduação em Engenharia Oceânica, Universidade Federal do Rio Grande. Rio Grande, RS. 2014. 98 p. 